\title{
Distinct Temporal Coordination of Spontaneous Population Activity between Basal Forebrain and Auditory Cortex
}

\author{
Josue G. Yague, Tomomi Tsunematsu and Shuzo Sakata* \\ Strathclyde Institute of Pharmacy and Biomedical Sciences, University of Strathclyde, Glasgow, United Kingdom
}

The basal forebrain (BF) has long been implicated in attention, learning and memory, and recent studies have established a causal relationship between artificial BF activation and arousal. However, neural ensemble dynamics in the BF still remains unclear. Here, recording neural population activity in the BF and comparing it with simultaneously recorded cortical population under both anesthetized and unanesthetized conditions, we investigate the difference in the structure of spontaneous population activity between the BF and the auditory cortex (AC) in mice. The AC neuronal population show a skewed spike rate distribution, a higher proportion of short ( $\leq 80 \mathrm{~ms}$ ) inter-spike intervals (ISIs) and a rich repertoire of rhythmic firing across frequencies. Although the distribution of spontaneous firing rate in the BF is also skewed, a proportion of short ISls can

OPEN ACCESS

Edited by:

Michael E. Hasselmo Boston University, United States

Reviewed by:

Heiko J. Luhmann, Johannes Gutenberg-Universität Mainz, Germany Jason William Middleton, LSU Health Sciences Center New

Orleans, United States

${ }^{*}$ Correspondence: Shuzo Sakata shuzo.sakata@strath.ac.uk

Received: 01 May 2017 Accepted: 31 August 2017 Published: 14 September 2017

Citation:

Yague JG, Tsunematsu T and Sakata S (2017) Distinct Temporal Coordination of Spontaneous Population Activity between Basal Forebrain and Auditory Cortex. Front. Neural Circuits 11:64. doi: 10.3389/fncir.2017.00064 be explained by a Poisson model at short time scales $(\leq 20 \mathrm{~ms})$ and spike count correlations are lower compared to AC cells, with optogenetically identified cholinergic cell pairs showing exceptionally higher correlations. Furthermore, a smaller fraction of BF neurons shows spike-field entrainment across frequencies: a subset of BF neurons fire rhythmically at slow $(\leq 6 \mathrm{~Hz})$ frequencies, with varied phase preferences to ongoing field potentials, in contrast to a consistent phase preference of AC populations. Firing of these slow rhythmic BF cells is correlated to a greater degree than other rhythmic BF cell pairs. Overall, the fundamental difference in the structure of population activity between the AC and $\mathrm{BF}$ is their temporal coordination, in particular their operational timescales. These results suggest that BF neurons slowly modulate downstream populations whereas cortical circuits transmit signals on multiple timescales. Thus, the characterization of the neural ensemble dynamics in the BF provides further insight into the neural mechanisms, by which brain states are regulated.

Keywords: acetylcholine, neural ensemble, optogenetics, neural oscillations, neural coding, brain state

\section{INTRODUCTION}

Neural population activity has been characterized by measuring several metrics over the past decades. For example, sparseness of cortical population activity has been shown to vary across cell types (Sakata and Harris, 2009; O'Connor et al., 2010; Barth and Poulet, 2012; Harris and MrsicFlogel, 2013), and a skewed log-normal distribution of firing rates has been repeatedly shown in 
cortical and hippocampal neurons (Hirase et al., 2001; Hromadka et al., 2008; Peyrache et al., 2012; Mizuseki and Buzsaki, 2013; Buzsaki and Mizuseki, 2014), imposing biophysical constraints on neural population activity. Correlated spiking is also well documented (Averbeck et al., 2006; Cohen and Kohn, 2011; Doiron et al., 2016) although the functions and underlying mechanisms of such correlated firing are still to be determined (Renart et al., 2010; Litwin-Kumar and Doiron, 2012; Middleton et al., 2012; Mochol et al., 2015; Doiron et al., 2016). Cortical neurons also show rhythmic firing on multiple timescales (Buzsaki and Draguhn, 2004; Schroeder and Lakatos, 2009) and such a rhythmic firing has been implicated in a communication strategy among cortical areas (Buzsaki, 2010; Fries, 2015). However, it is still unclear to what extent such firing regimes can be generalized to other brain areas. Although intensive efforts have been made recently to uncover the structural and functional principles of cortical circuit organization (Kasthuri et al., 2015; Markram et al., 2015; Hawrylycz et al., 2016), it is also important to elucidate any fundamental difference in neural ensemble dynamics between the cortex and other subcortical regions.

The basal forebrain (BF) is a crucial subcortical complex (Mesulam et al., 1983; Woolf, 1991; Zaborszky and Duque, 2003; Zaborszky et al., 2012; Lin et al., 2015; Ballinger et al., 2016). While it consists of multiple "cholinergic" nuclei (Mesulam et al., 1983; Woolf, 1991), BF cells are heterogeneous in terms of molecular, morphological, and electrophysiological properties (Zaborszky and Duque, 2003; Lee et al., 2005; Zaborszky et al., 2012; McKenna et al., 2013; Lin et al., 2015; Xu et al., 2015; Ballinger et al., 2016). While neurodegeneration in the BF is associated with Alzheimer's disease (Whitehouse et al., 1982; Ballinger et al., 2016), the BF has long been implicated in cortical desynchronization, plasticity, arousal, attention, learning and memory (Everitt and Robbins, 1997; Kilgard and Merzenich, 1998; Zaborszky and Duque, 2003; Weinberger, 2004; Hasselmo and Sarter, 2011; Sakata, 2016). Manipulations of BF neurons modulate cortical and behavioral states in a cell type-specific manner (Kalmbach et al., 2012; Pinto et al., 2013; Eggermann et al., 2014; Anaclet et al., 2015; Kim et al., 2015; Xu et al., 2015; Zant et al., 2016), consistent with the notion that the BF causally regulates arousal. Recent studies have also uncovered links to other cognitive domains, such as decision making and outcome expectations (Lin and Nicolelis, 2008; Hangya et al., 2015; Lin et al., 2015). Thus, much information has been accumulated about the BF, but little is known about how BF neurons act as a population to regulate brain states. Following pioneering works (Lin et al., 2006; Tingley et al., 2015), Dan and colleagues recently investigated cell type-specific activity during both spontaneous and task-related behavior (Harrison et al., 2016). However, BF population activity at high temporal resolution still remains largely unexplored, and in particular it has not been systematically compared with that of cortical population activity.

In the present study, we performed in vivo electrophysiological recording in both the mouse $\mathrm{BF}$ and auditory cortex $(\mathrm{AC})$, which is a well-characterized cortical area with respect to the structure of neural population activity (Sakata and Harris, 2009, 2012; Harris and Mrsic-Flogel, 2013; Kayser et al., 2015; Sakata, 2016), and to which the anatomical and functional relation of the BF has been investigated (Kilgard and Merzenich, 1998; Weinberger, 2004; Froemke et al., 2007; Chavez and Zaborszky, 2016; Nelson and Mooney, 2016). Comparing the structure of neural spontaneous activity between the $\mathrm{BF}$ and $\mathrm{AC}$, here we report that the temporal coordination of AC population activity is highly structured whereas BF populations show less coordination. Our results highlight the importance of comparisons of neural population activity in different brain regions to determine biophysical constraints on the timescale of population activity.

\section{MATERIALS AND METHODS}

\section{Animals}

A total of 32 transgenic mice expressing channelrhodopsin 2 (ChR2) in either cholinergic (22 males, 8 females; ChAT-IRESCre::Ai32) (ChAT-IRES-Cre, JAX006410; Ai32, JAX012569) or parvalbumin (2 females; PV-IRES-Cre::Ai32) (PV-IRES-Cre, JAX008069) positive neurons were used in this study. In PVIRES-Cre::Ai32 mice, only AC recording $(n=2)$ was included in the present study. Experiments were performed in accordance with the United Kingdom Animals (Scientific Procedures) Act of 1986 Home Office regulations and approved by the Home Office (PPL 70/8883).

\section{In Vivo Electrophysiology}

We carried out a total of 48 in vivo electrophysiological recordings under urethane anesthesia ( $\left.n_{\text {anesthetized }}=27\right)$ or unanesthetized head-fixed condition $\left(n_{\text {unanesthetized }}=21\right)$.

\section{Surgical Procedures for Experiments under Anesthesia}

Animals were anesthetized with $1.5 \mathrm{~g} / \mathrm{kg}$ urethane. Lidocaine ( $2 \%, 0.1-0.3 \mathrm{mg})$ was also administered subcutaneously at the site of incision. Two bone screws were fixed in the skull, one in the frontal region ( $\mathrm{AP}+3 \mathrm{~mm}, \mathrm{ML}+2 \mathrm{~mm}$ from bregma) used as an electrode for cortical electroencephalograms (EEGs) and one on the cerebellum as a ground and a reference. A craniotomy on the left hemisphere $(\mathrm{AP}+1$ to $-1 \mathrm{~mm}, \mathrm{ML}+1$ to $+3 \mathrm{~mm}$ from bregma) was performed to access the BF. For the auditory cortical recording, another craniotomy on the left hemisphere (AP -2 to $-4 \mathrm{~mm}$, ML $4-4.5 \mathrm{~mm}$ from bregma) was performed. Body temperature was maintained at $37^{\circ} \mathrm{C}$ with a feedback temperature controller (40-90-8C, FHC).

\section{Surgical Procedures for Experiments in Unanesthetized Head-Fixed Condition}

Animals were anesthetized with isoflurane (1-1.5\%). Lidocaine (2\%, $0.1-0.3 \mathrm{mg})$ was also administered subcutaneously at the site of incision. To provide analgesia after the surgery, Carprofen (Rimadyl, $5 \mathrm{mg} / \mathrm{kg}$ ) was administered intraperitoneously. A head-post was attached on the skull by implanting two frontal bone screws (AP $+3 \mathrm{~mm}$, ML $2 \mathrm{~mm}$ from bregma) one of them used for EEG recording. Another two screws were implanted on the cerebellum, one of them used as a ground and a reference. 
Then, a pair of nuts was attached with dental cement as a head-post. After the head-post surgery the animals were left to recover for at least 5 days. During the acclimation period, the animals were placed in a head-fixed apparatus (SR-8N-S, Narishige), with holding the head-post securely and placing the animal into an acrylic tube. This procedure was continued for at least 5 days, during which the duration of head-fixed was gradually extended from 15 to $60 \mathrm{~min}$. During this period, the animals were also exposed to the sound stimulation in the same manner as the actual electrophysiological recording (see below). A day after this acclimation period, the animals were anesthetized with isofluorane and a craniotomy to access the BF was performed. In some experiments, we carried out a second craniotomy to expose the primary $\mathrm{AC}$ in order to carry out a simultaneous $\mathrm{BF}$ and $\mathrm{AC}$ recording. In the following day, the animals were placed in the head-fixed condition to carry out the unanesthetized electrophysiological recording.

\section{Electrophysiological Recordings}

Recording procedures are described elsewhere (McAlinden et al., 2015; Sakata, 2016; Scharf et al., 2016). Briefly, all electrophysiological recordings were performed in a singlewalled acoustic chamber (MAC-3, IAC Acoustics) with the interior covered by 3 inches of acoustic absorption foam. After the surgical procedures described above, a 32 channel silicon optrode (A1 × 32-Poly3-10 mm-50-177-A32OA or A1 × 32Poly2-10 mm-50s-177-A32OA, NeuroNexus Technologies) was inserted slowly $(2-5 \mu \mathrm{m} / \mathrm{sec})$ with a motorized manipulator (DMA-1511, Narishige) into the BF $(4.0-5.0 \mathrm{~mm}$ from the cortical surface) at different rostro-caudal and medio-lateral locations. A second 32 channel silicon probe $(\mathrm{A} 1 \times 32$-Poly210 mm-50s-177-A32, NeuroNexus Technologies) was inserted using a manual micromanipulator (SM-25A, Narishige) for AC recordings (800 - $1000 \mu \mathrm{m}$ from the cortical surface). Both probes were inserted perpendicularly with respect to the cortical surface. The location of the electrode in AC was assessed by evaluating the local field potential (LFP) and multiunit activities (MUA) in response to white noise stimulation, which was generated digitally (sampling rate $97.7 \mathrm{kHz}$, TDT, Tucker-Davis Technologies) and delivered in free-field through a calibrated electrostatic loud-speaker (ES1) located $\sim 15 \mathrm{~cm}$ in front of the animal.

Broadband signals were amplified (HST/32V-G20 and PBX3, Plexon or RHD2132, Intan Technologies, LLF) relative to a screw anchored in the cerebellar bone and were digitized at $20 \mathrm{kHz}$ (PXI, National Instruments, or RHD2132 and RHD2000, Intan Technologies, LLC). Optical stimulation (see below) was applied during the probe penetration in order to identify cholinergic neurons in the $\mathrm{BF}$. The recording session was initiated > 30 min after the probe was inserted to its target depth, to allow for signal stabilization. A typical recording session consisted of a baseline recording of at least $5 \mathrm{~min}$ of spontaneous activity, followed by an optical stimulation protocol and then another baseline of spontaneous activity. During some of recordings, we also played acoustic stimuli, but the results during sound presentations are not reported in the present study.

\section{Optogenetic Experiments}

Detailed procedures are described in previous studies (McAlinden et al., 2015; Scharf et al., 2016). Briefly, blue light (450 or $470 \mathrm{~nm}$, PlexBright, Plexon) was delivered through a fiber optic on the silicon probe. The light output at tip of the probe was measured with a constant long ( $>1 \mathrm{~s})$ light pulse before probe insertion and was $59.8 \pm 17.8 \mathrm{~mW} / \mathrm{mm}^{2}$ (mean $\pm \mathrm{SD}$ ). Because the first spike latency of BF cholinergic neurons is known to be varied and slow (Unal et al., 2012), we applied optical stimulation for 50 or $100 \mathrm{~ms}$ at $2 \mathrm{~Hz}$ rate with up to 200 repetitions. However, to reduce the confounding effect of heating (McAlinden et al., 2013; Stujenske et al., 2015; Scharf et al., 2016) and indirect activation, we assessed neural activity only with $50 \mathrm{~ms}$ stimulations.

\section{Histology}

For verification of silicon probe tracks, the rear of probes was painted with DiI ( $\sim 10 \%$ in ethanol, D282, Molecular Probes) before probe insertion. After electrophysiological experiments, animals were perfused transcardially with physiological saline followed by $4 \%$ paraformaldehyde/ $0.1 \mathrm{M}$ phosphate buffer, $\mathrm{pH}$ 7.4. After an overnight post-fixation in the same fixative and cryoprotection with $30 \%$ sucrose in phosphate buffered saline (PBS), brains were cut into $100 \mu \mathrm{m}$ coronal sections with a sliding microtome (SM2010R, Leica) and placed in PBS. The sections were mounted on gelatin-coated slides and cover-slipped with antifade solutions and the area of the electrode was photographed using a fluorescence microscope. Based on a pattern of ChR2EYFP expression, we assessed whether the silicon probe was located in the BF. For a further assessment, sections were also Nissl stained (1\% w/v Cresyl Violet plus 1\% v/v Glacial Acetic Acid in $\mathrm{dH}_{2} \mathrm{O}$ ) to determine sub-nuclei of the BF.

To localize cholinergic neurons in the BF, immunohistochemistry was also performed with some sections. They were incubated with a blocking solution (10\% normal donkey serum, NDS, in $0.5 \%$ Triton $\mathrm{X}$ in PBS, PBST) for $1 \mathrm{~h}$ at room temperature followed by incubating primary antibodies (anti-ChAT, 1:200, AB114P, Millipore) in 3\% NDS in PBST at $4^{\circ} \mathrm{C}$ overnight. After washing, sections were incubated with secondary antibodies (donkey anti-boat Alexa Fluor 568, 1:1000, A11057, Life Science Technologies) for $2 \mathrm{~h}$ at room temperature. After washing, sections were mounted on gelatin-coated slides and cover-slipped with antifade solution.

\section{Data Analysis}

Data analysis was performed offline using MATLAB (Mathworks) or freely available software. To extract local field potentials (LFPs), a lowpass filter $(<100 \mathrm{~Hz})$ was applied and signals were downsampled to $1 \mathrm{kHz}$. To compute power spectral density, Chronux Toolbox ${ }^{1}$ was used. For spike sorting, the Klusta package (Rossant et al., 2016) was used. This spike sorting process consisted of a semi-automatic process with automatic spike detection and clustering followed by manual clustering, which can reduce spike sorting errors: (1) commission error where spikes belonging to different neurons are clustered

\footnotetext{
${ }^{1}$ http://chronux.org/
} 
together, for example due to synchronous spiking by nearby neurons, and (2) omission errors where not all spikes emitted by a single neuron are grouped together, such as burst firing (Harris et al., 2000; Rossant et al., 2016). After spike sorting, the quality of single units was further assessed by measuring isolation distance (Schmitzer-Torbert et al., 2005). The inclusion criteria for single units in the present study were $\geq 50$ isolation distance and $\geq 0.1 \mathrm{~Hz}$ spontaneous firing. It might be argued that $\geq 50$ isolation distance is apparently too conservative as $\geq 20$ isolation distance has been often set. However, because we used all 32 channels for spike sorting and isolation distance increases depending on the number of features used, we set the inclusion criteria as $\geq 50$ isolation distance.

\section{Cell Type Classifications}

For BF cells, the optogenetic tagging method was applied (see above). The statistical significance of spike counts in a $50 \mathrm{~ms}$ time window during optical stimulations was assessed and compared with those in a pre-stimulus time window $(50 \mathrm{~ms})$ by performing Bonferroni corrected rank sum test. A p-value of less than 0.05 was recognized as being statistically significant, and significant cells were then categorized as modulated cells. Modulated cells with a significant increase in spike counts were categorized as cholinergic neurons. In the present study, only one neuron showed significant reduction in spike counts, and was categorized as a non-cholinergic neuron within the non-modulated neuron group.

For AC cells, a conventional classification approach was used based on average spike waveforms (Sakata and Harris, 2009, 2012; Sakata, 2016). Briefly, trough to peak time and spike width at $20 \%$ of depth were computed from the averaged spike waveforms of each single unit. A threshold of $0.4 \mathrm{~ms}$ for trough to peak time and 0.3 ms spike width at $20 \%$ of depth was used to classify narrow spiking (NS) or broad spiking (BS) cells.

To estimate the distance of recorded neurons (Figure 6), the channel showing the maximum trough to peak amplitude was recognized as the putative somatic position of recorded neurons. Then the distance was determined by the silicon probe design. This estimate was based on observations in previous literature (Henze et al., 2000), in which the amplitude of extracellular spike waveforms becomes the largest at the perisomatic area.

\section{Firing Parameter Estimation}

Spontaneous firing rate was estimated by counting the total number of spikes during the spontaneous period across single units. To assess a temporal pattern of spiking activity, a proportion of inter-spike intervals (ISIs) with a particular duration $T$ (e.g., $\leq 10 \mathrm{~ms}$ ) (called ISI $_{\leq \mathrm{T}}$ ) was computed. For a control for this analysis, we took the estimated spontaneous firing rate for each cell to generate a Poisson spike train to compute the same index.

\section{Spike Count Correlations}

To compute spike count correlations during spontaneous activity, we took the following approach: for neuron $i$, the number of spikes at time $t$ was counted as $n_{\mathrm{i}}(t)$ in each bin (bin size $=1 \mathrm{~ms}$ ) convoluted by a Gaussian kernel of standard deviation $T(T=100 \mathrm{~ms})$. Then spike count correlations between the activity of neuron $i n_{\mathrm{i}}(t ; T)$ and neuron $j n_{\mathrm{j}}(t ; T)$ were computed

$$
r_{\mathrm{ij}}(T)=\frac{\operatorname{Cov}\left(n_{\mathrm{i}}, n_{\mathrm{j}}\right)}{\sqrt{\operatorname{Cov}\left(n_{\mathrm{i}}, n_{\mathrm{i}}\right) \operatorname{Cov}\left(n_{\mathrm{j}}, n_{\mathrm{j}}\right)}}
$$

where $\operatorname{Cov}\left(n_{\mathrm{i}}, n_{\mathrm{j}}\right)$ is the covariance between the activity of the two neurons. We used a MATLAB function, corrcoef.

Because neuronal activity is non-stationary and thus can co-vary on a wide range of time-scales, making the evaluation of temporal spike coordination challenging (Grün, 2009), we synthetized surrogate data which maintains a specified mean firing rate for each neuron and specified population rate distribution by using the raster marginals model (Okun et al., 2012). First, the original spike train was binned at $1 \mathrm{~ms}$. Then a binary matrix ( 0 , no spike; 1 , spike) was constructed with one column for each time bin and one row for each neuron. To synthetize surrogate data, random 2-by-2 submatrices were repeatedly chosen with each row and column of the submatrix containing a 0 and 1 . The positions of 0 s and 1 s were exchanged in the submatrix, which leaves the summed values of each row and column identical. Compared with a shuffling method (Grün, 2009; Renart et al., 2010) which can preserve the temporal dynamics of population rate of the original data depending on the jittering window size, the raster marginal model discards the temporal structure of the spike trains, with a specified mean firing rate for each single unit and specified population rate distribution.

\section{Spike-LFP Phase Analysis}

Detailed procedures are described elsewhere (Kayser et al., 2015). Briefly, LFP signal during the spontaneous period was extracted for each single unit. To avoid spurious estimates of spike entrainment to LFPs at high frequency bands, LFP signals were taken from spatially distinct channels that did not contain any spike signals from a given single unit. A Kaiser finite impulse response filter (sharp transition bandwidth of $1 \mathrm{~Hz}$, pass-band ripple of $0.01 \mathrm{~dB}$ and stop-band attenuation of $50 \mathrm{~dB}$ with forward and backward filtering using MATLAB 'filtfilt' function) was used to derive band-limited signals in different frequency bands. In the present study, we assessed the following bands: [2-3], [4-5], [6-8], [8-10], [10-15], [15-20], [20-25], [25-30], [30-40], [40-50], [60-70], [80-90], and [100-110] Hz. The instantaneous phase of each band was estimated from the Hilbert transform and spike phase was computed. To quantify the relationship between spikes and LFP phase, we calculated the percentage of spikes elicited in each phase bin. The rate modulation was defined as the percentage in the preferred bin (the bin with maximal percentage) minus that in the opposite bin (the bin $180^{\circ}$ apart). Rayleigh's test for non-uniformity of circular data was performed to assess the statistical significance $(p<0.01)$ of the non-uniformity of the spike-LFP phase distribution using CircStats Toolbox.

Out of all BF neurons, neurons which showed significant modulation at any frequency band were recognized as rhythmic $\mathrm{BF}$ cells. Of these, BF neurons which showed significant modulation at [2-3], [4-5], or [6-8] Hz were recognized as "slow 
rhythmic BF cells." In Figure 7, spike count correlations were computed in slow rhythmic cell pairs, slow vs. non-slow rhythmic cell pairs, and non-slow rhythmic cell pairs, as described above. In addition, correlations were computed using Gaussian kernels of different standard deviations $(5,10,25,50,75,100,150$, $200 \mathrm{~ms})$.

\section{Statistical Analysis}

Data were presented as mean \pm SEM unless stated. All confirmatory analyses were conducted using MATLAB: Student's $t$-test was carried out in Figures 3D,E. One-way ANOVA with post-hoc Tukey's honest significant difference (HSD) test was carried out in Figures 4A,B,D, 5A, 7. Two-way ANOVA with post hoc HSD test was carried out in Figures 4A-C, 5C,E. Due to the highly skewed distribution of burstiness (Figures 4D-J), Kruskal-Wallis one-way ANOVA with post hoc Bonferroni corrected Wilcoxon rank sum test (Figures 4D,K) and Wilcoxon rank sum test (Figures $\mathbf{4 E}-\mathbf{J}$ ) were carried out. Chi-square goodness-of-fit test was carried out to compare two distributions in Figures 6B,D. To estimate effect size, Hedges' $g$ was computed using Measures of Effect Size Toolbox.

\section{RESULTS}

\section{Database}

To compare population activity between the $\mathrm{AC}$ and $\mathrm{BF}$, we performed in vivo electrophysiological experiments in both urethane anesthetized and head-restrained unanesthetized mice. Of 48 recordings from 32 animals, we monitored population activity from the AC in 23 experiments $\left(n_{\text {anesthetized }}=8\right.$; $n_{\text {unanesthetized }}=15$ ) and from the $\mathrm{BF}$ in 44 experiments $\left(n_{\text {anesthetized }}=27 ; n_{\text {unanesthetized }}=17\right)$. Electrodes were positioned throughout the BF nuclei, including the basal nucleus of Meynert, nucleus of the horizontal limb of the diagonal band, substantia innominata, extended amygdala, and ventral pallidum (Figure 1). Of 44 experiments, eight $\mathrm{BF}$ experiments $\left(n_{\text {anesthetized }}=5\right.$; $n_{\text {unanesthetized }}=3$ ) were excluded due to the miss-positioning of the electrode following histological assessment. Of the remaining, 15 experiments ( $n_{\text {anesthetized }}=5 ; n_{\text {unanesthetized }}=10$ ) were simultaneous recording of both the BF and AC (Figure 2A). Power spectral density of cortical field potentials differed between the anesthetized and unanesthetized conditions (Figures 2B,C), indicating that both experimental conditions represent different brain states. Under urethane anesthesia, we observed UP and DOWN states in the mouse AC (Figure 2A) as previously reported in the AC and other cortical areas (Steriade et al., 1993; Steriade, 2001; Luczak et al., 2007; Curto et al., 2009; Sakata and Harris, 2009, 2012; Reyes-Puerta et al., 2016; Sakata, 2016). Although population activity could be assessed only during UP states to compare with that during a desynchronized state (Renart et al., 2010; Sakata and Harris, 2012; Sakata, 2016), in the present study we treated the anesthetized state as a single condition.

\section{Cell Type Classification}

We isolated > 300 single units in each area: a total of 357 $\mathrm{BF}$ cells $\left(n_{\text {anesthetized }}=285 ; n_{\text {unanesthetized }}=72\right)$ and $353 \mathrm{AC}$ cells $\left(n_{\text {anesthetized }}=130 ; n_{\text {unanesthetized }}=223\right.$ ) were isolated (see Materials and Methods).

We further classified cell types: in the BF, we adopted an optogenetic tagging approach in which channelrhodopsin2 (ChR2) was expressed in cholinergic neurons (Figure 3A) and spikes in ChR2-positive neurons were elicited by optical stimulation during electrophysiological recordings (Figure 3B). Optical stimulation significantly modulated 24 out of 357 isolated BF neurons (Figure 3C). Of these modulated cells, only one cell showed suppression. Therefore, we classified optically excited cells as cholinergic neurons $(n=23)$ and other cells, including the suppressed cell, as non-cholinergic neurons $(n=334)$. We assessed the distortion of average spike waveforms between optically induced spikes and spontaneous ones in both cholinergic and non-cholinergic neurons by computing Pearson's correlation coefficient. We did not find any significant difference between cholinergic and non-cholinergic neurons $(p=0.35$, t-test) (Figure 3D). However, we found that the spike width (trough to peak time) of cholinergic neurons significantly differs from that of non-cholinergic neurons $(p<0.0005, t$-test $)$ (Figure 3E), suggesting physiologically different cell classes. In the $\mathrm{AC}$, we classified neurons into two types based on their spike waveforms (Figure 3F). This resulted in 300 broadspiking (BS) and 53 narrow-spiking (NS) cells, many of which may be parvalbumin-positive interneurons (Madisen et al., 2012).

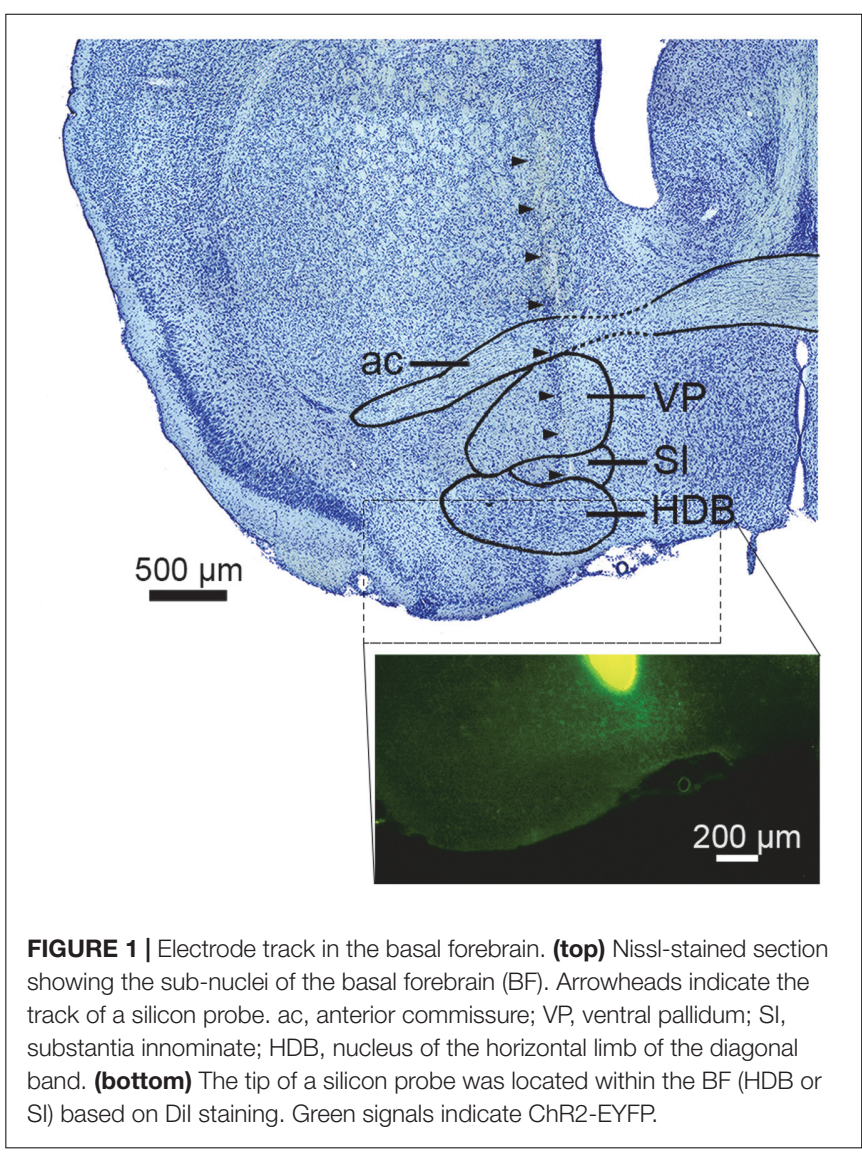




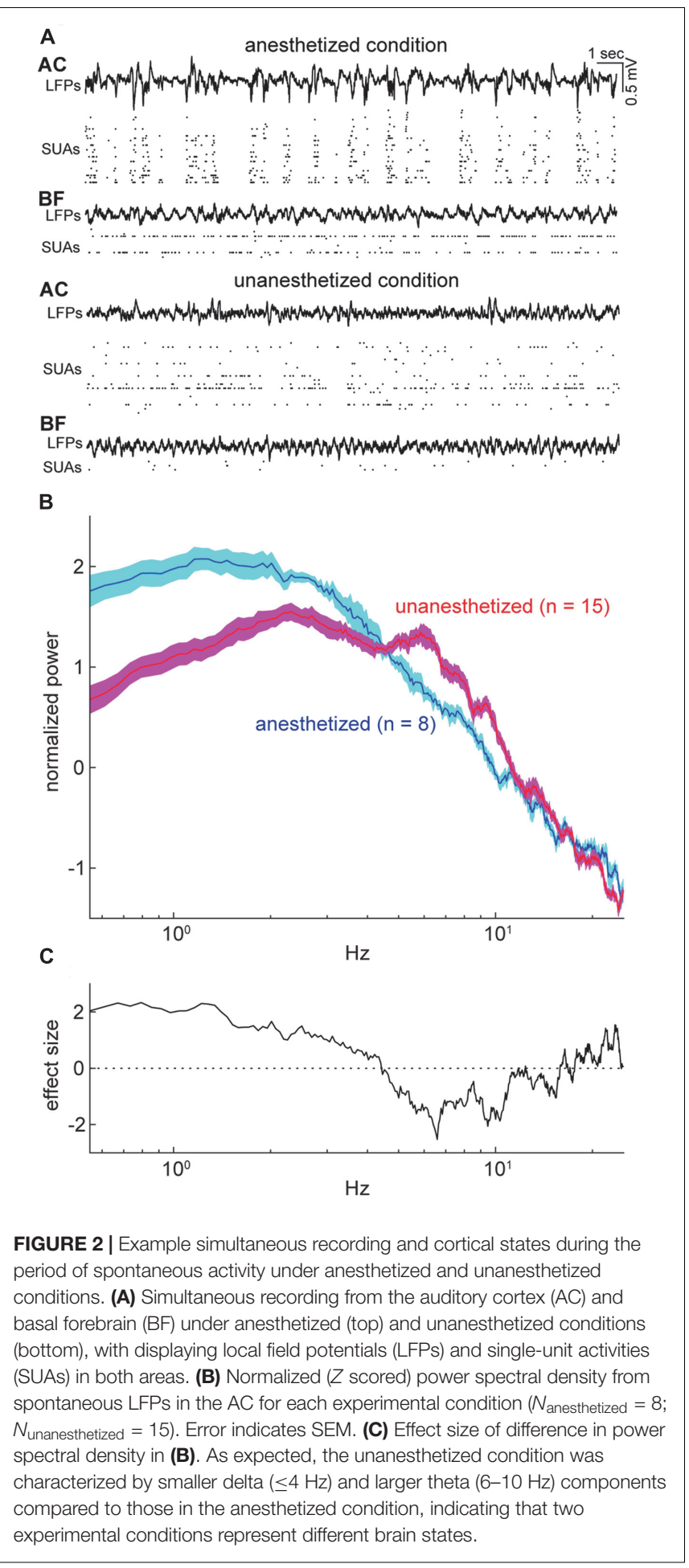

\section{Similarities and Differences in Spontaneous Firing Rate and Inter-spike Intervals between BF and AC Neurons}

To assess basic firing properties of $\mathrm{BF}$ and $\mathrm{AC}$ populations at the single unit level, we first compared spontaneous firing rate (Figure 4) between two areas. As shown in Figure 4A, the distribution of firing rate was skewed in both areas. To statistically compare mean firing rates in log scale between two areas under two conditions (anesthetized and unanesthetized), a two-way ANOVA was carried out: although the effect of condition was not significant $\left(F_{1,706}=0.05, p=0.83\right)$, the effect of brain area was significant $\left(F_{1,706}=6.31, p=0.012\right)$ and there was a significant interaction of condition and area $\left(F_{1,706}=15.16\right.$, $p=0.0001)$. More specifically, mean firing rate in the $\mathrm{BF}$ was higher than that in the AC [ $p<0.0001$, post hoc Tukey's honest significant difference (HSD) test] under anesthesia (Figure 4A). Both areas showed changes in firing rate depending on conditions $(p<0.05)$, but in opposite ways. We also examined the effect of cell-type and condition on mean firing rate and their interaction in each area (Figures $4 \mathbf{B}, \mathbf{C}$ ). No significant effect $\left(\mathrm{BF}: F_{1,353}=1.16, p_{\text {cell-type }}=0.28 ; F_{1,353}=0.56\right.$, $\left.p_{\text {condition }}=0.45\right)\left(\mathrm{AC}: F_{1,349}=0.22, p_{\text {cell-type }}=0.63\right.$; $\left.F_{1,349}=2.45, p_{\text {condition }}=0.11\right)$ nor interaction $\left(\mathrm{BF}: F_{1,353}=0.03\right.$, $p=0.86$ ) (AC: $F_{1,349}=0.79 ; p=0.37$ ) was found. Effect size between cholinergic and non-cholinergic neuron firing rates was 0.47 and 0.31 in anesthetized and unanesthetized conditions, respectively. Thus, although no significant cell type specificity was found, the skew distribution of mean firing rate was common in both areas and experimental conditions.

Next, to investigate a temporal structure of firing at the level of individual neurons and its dependency of timescales, we firstly computed the proportion of $\leq 10 \mathrm{~ms}$ inter-spike intervals $\left(\mathrm{ISI}_{\leq 10}\right)$ (Figures $\left.4 \mathrm{D}-\mathrm{J}\right)$. AC cells showed significantly higher ISI $_{\leq 10}$ compared to BF cells $(p<0.0005$, Kruskal-Wallis test with post hoc rank sum test) (Figure 4D). To check whether this result can be explained by a Poisson model, we generated a randomized spike train with the same mean firing rate, and then computed $\mathrm{ISI}_{\leq 10}$. There was a significant difference in the median of $\mathrm{ISI}_{\leq 10}$ between real and artificially generated spike trains in the AC $(p<0.0005)$, but not in the $\mathrm{BF}$ $(p=0.364)$, suggesting temporally structured firing in AC neurons and a random nature of $\mathrm{BF}$ cell firing in a short time scale. Intriguingly, this trend was not held at longer time scales ( $\geq 40 \mathrm{~ms}$ ) (Figure 4K). Experimental conditions (anesthetized or unanesthetized) significantly affected this assessment in the BF ( $p<0.0005$, rank sum test) (Figure 4E), but not in the AC $(p=0.09)$ (Figure $4 \mathrm{H})$. No statistically significant differences between cell types were observed (Figure 4F, $p=0.225$; Figure 4G, $p=0.311$; Figure 4I, $p=0.563$; Figure 4J, $p=0.261$ ). In sum, although no cell type specificity was found, AC neurons showed temporally organized firing even at short time scales compared to BF neurons at the single cell level.

\section{Differences in Spike Count Correlations between BF and AC}

Analysis at the single cell level suggests differences in the temporal structure of firing between the $\mathrm{AC}$ and $\mathrm{BF}$. To investigate the structure of neural firing at the population level, we first quantified temporal correlation between spike trains and asked whether there is any cell type specificity in co-firing. To this end, we computed spike count correlations across 

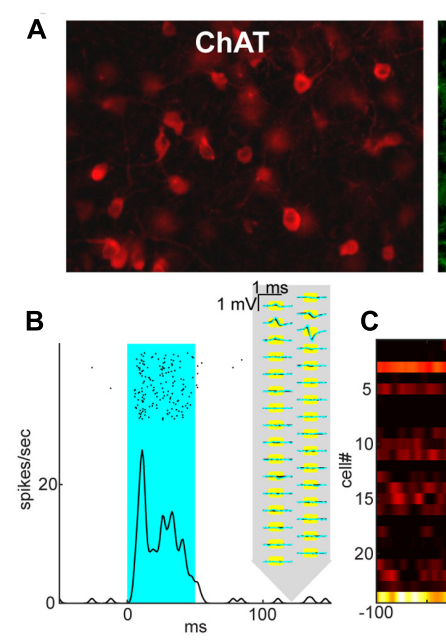

c
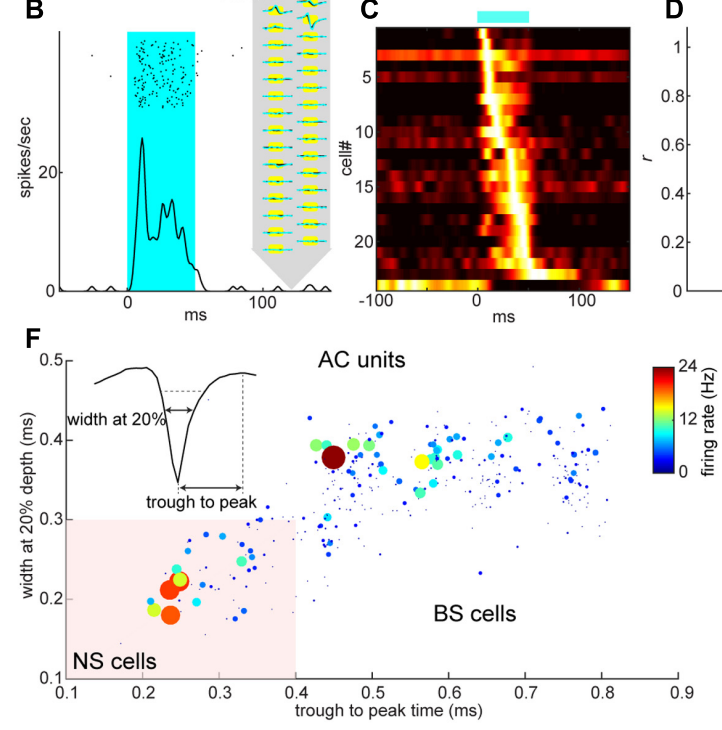
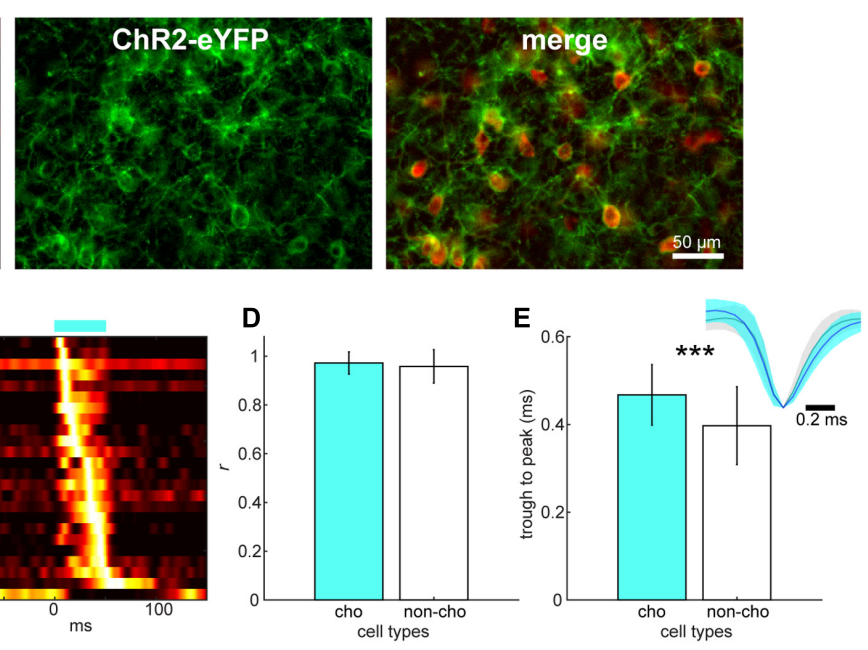

(1)

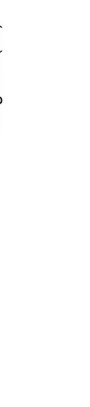

recorded neurons in each area (Figure 5). AC neurons showed higher correlations compared to BF neurons $\left(F_{3,74455}=11486\right.$, $p<0.0001$, two-way ANOVA with post hoc HSD test) whereas both populations showed significantly higher correlations than surrogate data, generated by the raster marginals model (Okun et al., 2012) $(p<0.0001)$ (Figure 5A). In the BF, optogenetically identified cholinergic cell pairs showed higher correlations than other pairs $\left(F_{5,30761}=1628, p<0.0001\right)$ (Figure 5B). Although the number of simultaneously recorded cholinergic cell pairs was very limited in the unanesthetized condition $(n=1)$, this tendency was held regardless of window size (Figures 5F-H). Effect size between cholinergic pairs and other pairs (vs. cho-non-cho pairs, 0.64; vs. non-cho-non-cho pairs, 0.91) was also larger than that between other pairs (0.22). In the AC, although we found statistically significant effect of pair types $\left(F_{5,43685}=5323, p<0.0001\right)$ (Figure $5 D$ ), effect size was small (BS-BS vs. BS-NS, 0.040; BS-BS vs. NS-NS, 0.14; BS-NS vs. NS-NS, 0.084). We also assessed an interaction between pair types and distance by separating each pair into two groups depending on estimated distance (local pairs, $\leq 150 \mu \mathrm{m}$; distal pairs,
$>150 \mu \mathrm{m}$ ) based on the depth profile of average spike waveforms. We found a significant interaction of pair types and distance in both the $\mathrm{BF}\left(F_{2,2791}=4.52, p<0.05\right.$, two-way ANOVA $)$ (Figure 5C) and $\mathrm{AC}\left(F_{2,3966}=3.03, p<0.05\right)$ (Figure 5E), with significant decrease in spike count correlations in some of distal pairs. In summary, spike trains of AC populations showed higher temporal correlation than those of BF populations. Within the $\mathrm{BF}$, cholinergic cell pairs showed higher correlations than non-cholinergic cell pairs.

\section{Differences in Spike-Field Entrainment between BF and AC}

To further investigate the temporal coordination between spiking and net synaptic activity in both the BF and AC, we examined to what extent spikes entrained to ongoing field potentials across different frequency bands $(2-100 \mathrm{~Hz}$ ) (Figure 6). A handful of $\mathrm{BF}$ neurons showed significant entrainment to slow components of LFPs (Figures 6A-C), suggesting rhythmic firing within the BF. As shown in Figure 6A, this example BF neuron clearly 

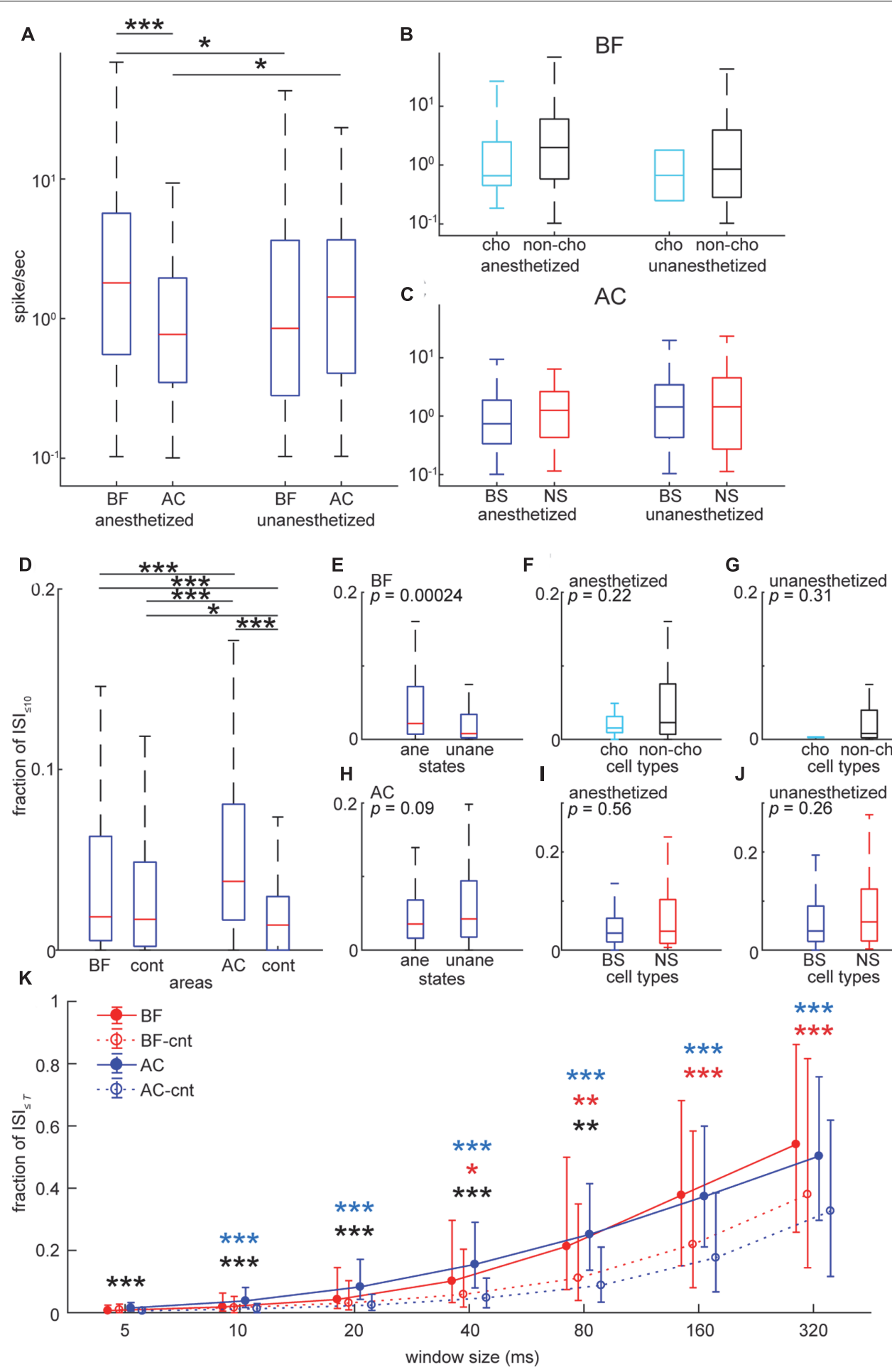

FIGURE 4 | Comparison of spontaneous firing rate and inter-spike intervals in the BF and $\mathrm{AC}$ at the single cell level. (A) Comparison of average spontaneous firing rate between the $\mathrm{BF}$ and $\mathrm{AC}$ in log scale. The effect of brain area was significant $\left(F_{1,706}=6.31, p=0.012\right)$ whereas the effect between anesthetized and non-anesthetized conditions was not significant $\left(F_{1,706}=0.045, p=0.83\right)$. The interaction of brain area and state was significant $\left(F_{1,706}=15.1, p=1.08 \mathrm{e}-04\right)$. ${ }^{* * *} p<0.0001 ; * p<0.05$ [post hoc honest significant difference (HSD) test]. (B) Comparisons of average spontaneous firing rate between cholinergic (cho) and non-cholinergic (non-cho) neurons in the BF. The effect of cell type $\left(F_{1,353}=1.15, p=0.28\right)$ or state $\left(F_{1,353}=0.56, p=0.45\right)$ was not significant. No significant interaction of cell type and state was observed $\left(F_{1,353}=0.029, p=0.86\right)$. (C) Comparisons of average spontaneous firing rate between BS and NS cells in the AC. The effect of cell type $\left(F_{1,349}=0.22, p=0.63\right)$ or state $\left(F_{1,349}=2.45, p=0.11\right)$ was not significant. No significant interaction of cell type and state was observed 
FIGURE 4 | Continued

$\left(F_{1,349}=0.78, p=0.37\right)$. (D) Comparison of $\mathrm{ISI}_{\leq 10}$ (a proportion of $\leq 10 \mathrm{~ms}$ inter-spike intervals) between the BF and AC. As a control, Poisson spike trains with the same spike rate distribution were included for statistical assessment. The effect of cell group was significant $\left(X^{2} 3,1416=123, p=1.46 \mathrm{e}-26\right.$, Kruskal-Wallis one-way ANOVA). ${ }^{*} p<0.005,{ }^{* *} p<0.0001$ (post hoc Bonferroni corrected Wilcoxon rank sum test). (E-J) Comparisons of ISI $\leq 10$ in the BF (E-G) and AC (H-J) in different cell types and states. P-values were derived from rank sum test. (K) Dependency of window size $T$ on $I S I_{\leq T}$ of $B F$ and $A C$ cells. Median is indicated with quartiles across cell groups. In control groups (BF-cnt and AC-cnt), Poisson spike trains were generated based on mean firing rate. For confirmatory analysis, Kruskal-Wallis one-way ANOVA was performed for each time window $T\left(5 \mathrm{~ms}, X^{2} 3,1416=76.3, p=1.89 \mathrm{e}-16 ; 10 \mathrm{~ms}, X^{2} 3,1416=76.3, p=1.85 \mathrm{e}-16 ; 20 \mathrm{~ms}, X^{2} 3,1416=122\right.$, $p=2.02 \mathrm{e}-26 ; 40 \mathrm{~ms}, X^{2}{ }_{3,1416}=150, p=2.27 \mathrm{e}-32 ; 80 \mathrm{~ms}, X^{2}{ }_{3,1416}=147, p=9.08 \mathrm{e}-32 ; 160 \mathrm{~ms}, X^{2} 3,1416=132, p=1.59 \mathrm{e}-28 ; 320 \mathrm{~ms}, X^{2} 3,1416=106$, $p=7.86 \mathrm{e}-23) .{ }^{*} p<0.05,{ }^{* *} p<0.005,{ }^{* * *} p<0.0005$ (post hoc Bonferroni corrected Wilcoxon rank sum test) (black, BF vs. AC; red, BF vs. BF-cnt; blue, AC vs. AC-cnt).
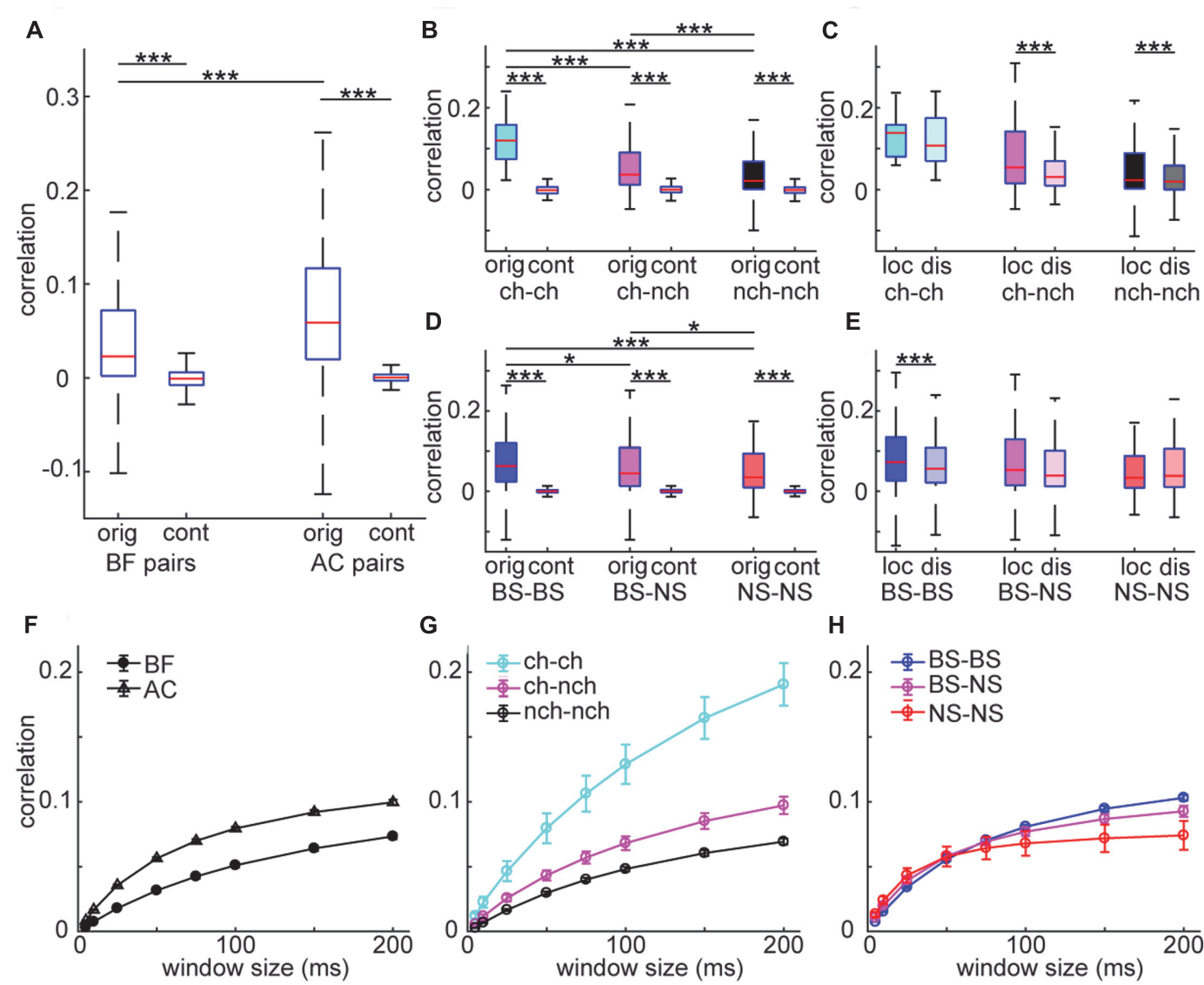

E

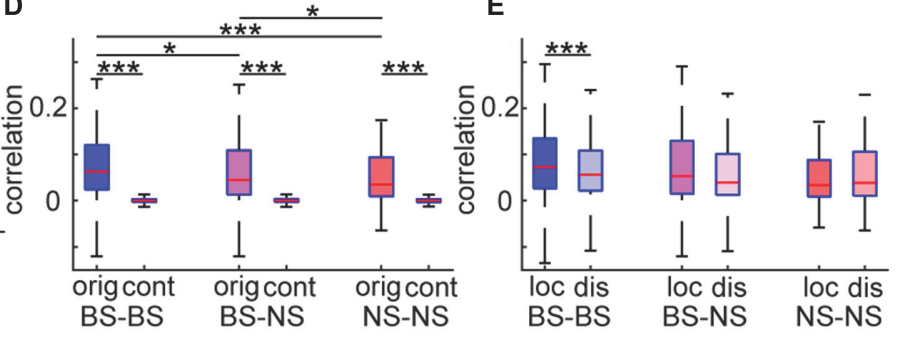

H

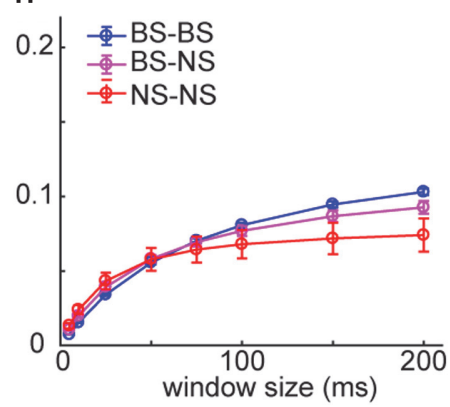

FIGURE 5 | Spike count noise correlations in BF and AC neuron pairs. (A) Comparison of correlations between BF and AC pairs. As a control (cont), surrogate data was generated by using the raster marginals model. The effect of pair was highly significant $\left(F_{3,74455}=1148, p<0.00001\right.$, one-way ANOVA). *** $p<0.00001$ (post hoc HSD test). orig, original correlations; cont, control. (B,D) Comparisons of correlations across different types of cell pairs in the BF (B) and AC (D), with control. The effect of pair type was significant in both the BF $\left(F_{5,30761}=1628, p<0.00001\right)$, and AC $\left(F_{5,43686}=5323, p<0.00001\right)$. Cholinergic cell pairs showed higher correlations compared to other pairs $(p<0.05) .{ }^{* * *} p<0.0001$ (post hoc HSD test within pair types). ch, cholinergic neurons; nch, non-cholinergic neurons. (C,E) Comparisons of correlation between local $(\leq 150 \mu \mathrm{m})$ and distal $(>150 \mu \mathrm{m})$ pairs across different types of cell pairs in the BF (C) and AC (E). In the BF (C), the effect of pair type was significant $\left(F_{2,2791}=16.6, p=6.56 \mathrm{e}-08\right.$, two-way ANOVA) whereas the effect of distance was not $\left(F_{1,2791}=3.68, p=0.054\right)$. A significant interaction of pair type and distance was observed $\left(F_{2,2791}=4.52, p=0.010\right)$. In the AC, no effect of pair type $\left(F_{2,3966}=1.34, p=0.25\right)$ or distance $\left(F_{1,3966}=1.16\right.$, $p=0.27$ ) was detected whereas a significant interaction of pair type and distance was observed $\left(F_{2,3966}=3.03, p=0.048\right) . * * * p<0.0001$ (post hoc HSD test within pair types). (F-H) Dependency of window size on spike count correlations. (F) Spike count correlations of BF and AC cell pairs across window sizes. (G) Spike count correlations of different cell pairs in the BF across window sizes. (H) Spike count correlations of different cell pairs in the AC across window sizes.

showed rhythmic firing at $2 \mathrm{~Hz}$, but not at $80 \mathrm{~Hz}$. Of 357 BF neurons, 163 cells (45.6\%) showed significant rhythmic modulations at any of analyzed frequency bands, but mainly at slow $(\leq 6 \mathrm{~Hz})$ frequencies $(n=140,39.2 \%)$. We did not find significant differences in the distribution of modulated cells across frequencies, either among cell types $(p=0.98$, chi-square goodness-of-fit test) or conditions (anesthetized vs. unanesthetized conditions) $(p=0.99)$. In addition, preferred phases at $2 \mathrm{~Hz}$ also varied across cells (Figure 6C). In contrast to $\mathrm{BF}$ populations, AC neurons showed highly structured 

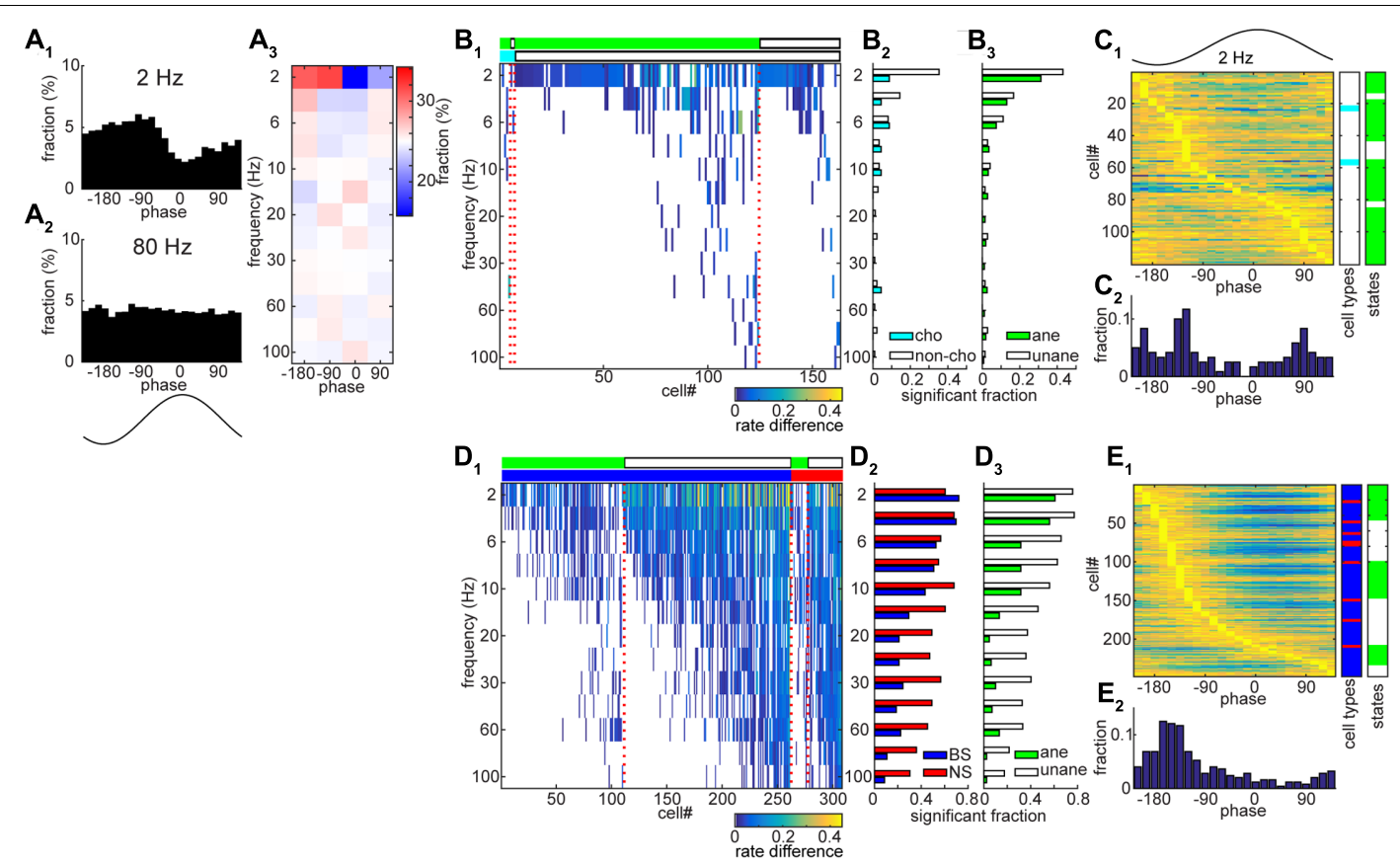

FIGURE 6 | Entrainment of spontaneous spike trains to rhythmic LPFs in the BF and AC. (A) An example of BF cell with showing rate modulation by slow (2 Hz) rhythmic LFPs. In $\left(\mathbf{A}_{\mathbf{1}}\right)$ and $\left(\mathbf{A}_{\mathbf{2}}\right)$, the fraction of spikes is shown as a function of LFP phase at a particular frequency band. In $\left(\mathbf{A}_{3}\right)$, phase bins were divided into four phases and the fraction of spikes was color-coded. (B) A summary of rate modulations across frequency bands in the BF. In ( $\left.\mathbf{B}_{1}\right)$, rate difference was defined as the difference in the fraction of spikes at between the peak phase and $180^{\circ}$ opposite phase. Rate difference was color-coded only at frequencies with significant modulation $(p<0.01$, Rayleigh's test). Different experimental conditions (light green, anesthetized; white, unanesthetized) and cell types (cyan, cholinergic cells; white, non-cholinergic cells) are shown in the top bars, respectively. In $\left(\mathbf{B}_{\mathbf{2}}, \mathbf{B}_{\mathbf{3}}\right)$ the fraction of cells which showed significant modulations was shown. No significant difference in the distribution was detected $\left(\mathbf{B}_{\mathbf{2}}, p=0.98 ; \mathbf{B}_{\mathbf{3}}, p=0.99\right.$, chi-square goodness-of-fit test). (C) Phase modulations of $\mathrm{BF}$ cells at $2 \mathrm{~Hz}$. The fraction normalized by the peak value was shown in $\left(\mathbf{C}_{\mathbf{1}}\right)$ with information about cell types and experimental conditions. In $\left(\mathbf{C}_{\mathbf{2}}\right)$ the distribution of peak phases was shown. (D) A summary of rate modulations across frequency bands in the AC. In $\left(\mathbf{D}_{1}\right)$, rate difference was color-coded. Different experimental conditions (light green, anesthetized; white, unanesthetized) and cell types (blue, BS cells; red, NS cells) are shown in the top bars, respectively. In $\left(\mathbf{D}_{\mathbf{2}}, \mathbf{D}_{3}\right)$ the fraction of cells which showed significant modulations was shown. A significant difference in the distribution was detected $\left(\mathbf{D}_{\mathbf{2}}, p=1.2 \mathrm{e}-23 ; \mathbf{D}_{\mathbf{3}}, p=6.0 \mathrm{e}-63\right)$. (E) Phase modulations of AC cells at $2 \mathrm{~Hz}$.

firing (Figures 6D,E), showing entrainment in $87.2 \%$ of cells $(308 / 353)$ at any of the frequency bands analyzed (Figure $\mathbf{6 D}_{\mathbf{1}}$ ). Notably there is a clear cell type specificity $(p<0.0001$, chisquare goodness-of-fit test), with a higher fraction of NS cells showing significant modulations at higher frequencies $(>10 \mathrm{~Hz})$ (Figure $6 \mathrm{D}_{2}$ ). A larger fraction of AC cells showed entrainment under the unanesthetized condition $\left(p<0.0001\right.$ ) (Figure $\mathbf{6 D}_{3}$ ). AC neurons showed a unimodal distribution of peak phase at $2 \mathrm{~Hz}$ (Figure 6E). Thus, the AC neuronal population shows temporally coordinated activities on multiple timescales whereas a smaller subset of BF neurons shows spike-field entrainment especially at slow $(\leq 6 \mathrm{~Hz})$ frequencies.

\section{Higher Spike Count Correlations of Slow Rhythmic BF Cells}

Finally, we examined whether coordinated spiking of the rhythmic BF neurons differs depending on their rhythmic modulations. To this end, we re-assessed spike count correlations between the rhythmic BF cells $(n=163)$ by dividing into two groups, slow $(\leq 6 \mathrm{~Hz})(n=140)$ and non-slow rhythmic cells $(n=23)$ (Figure 7$)$. Slow rhythmic BF cell pairs showed significantly higher correlations compared to other cell pairs in a $100 \mathrm{~ms}$ time window $\left(F_{2,801}=5.74, p=0.0033\right.$, twoway ANOVA). This was also the case in different time windows (75-200 ms). Thus, firing of slow rhythmic BF neurons was more correlated than that of other rhythmic BF neuron pairs.

\section{DISCUSSION}

The distribution of spontaneous firing rate was skewed in both the $\mathrm{AC}$ and $\mathrm{BF}$. However, BF populations showed firing statistics distinct from those of AC populations, with BF cells firing densely under anesthesia and showing less temporally structured firing at a short time scale ( $\leq 20 \mathrm{~ms})$, which is largely explained by a Poisson model. At the population level, BF cells showed lower spike count correlations, with the exception of cholinergic cell pairs, and less spike-field entrainment to LFPs, compared to AC cells. Overall, the fundamental difference in the structure of population activity between the AC and BF is their operational timescales. Given the modulatory nature of BF populations at a relatively slow timescale, less temporally coordinated firing may be appropriate to modulate downstream populations. Rather, cortical circuits should be recognized as 


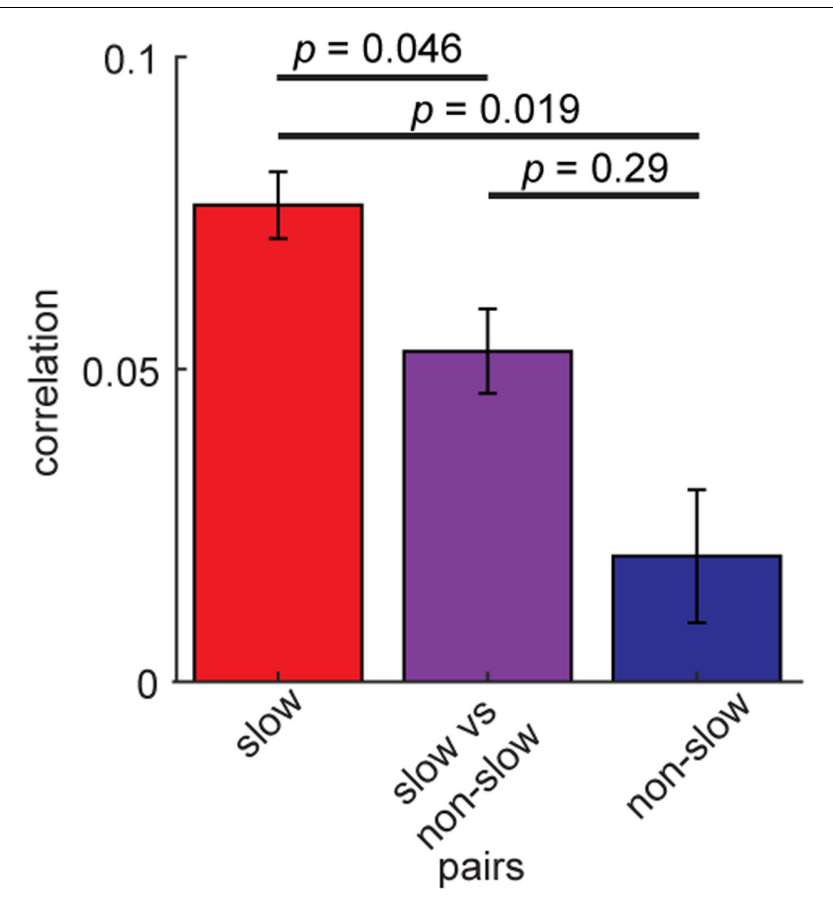

FIGURE 7 | Higher spike count correlations of slow rhythmic BF cells. Mean spike count correlations of slow $(\leq 6 \mathrm{~Hz})$ rhythmic BF cells $(n=140)$ and other rhythmic BF cells $(n=23)$. The effect of pair type was significant

$\left(F_{2,801}=5.74, p=0.0033\right.$, one-way ANOVA $)$. $P$-values of post hoc HSD test are shown. Errors indicate SEM.

a specialized neural circuit allowing signal transmission on multiple timescales.

The present study builds on previous studies in the BF (Lin et al., 2006; Tingley et al., 2015) by using an optogenetic tagging approach along with cortical ensemble recording. Similar optogenetic approaches have been recently used in the $\mathrm{BF}$ in vivo (Hangya et al., 2015; Xu et al., 2015). Although we identified a statistically significant difference in spike waveforms between cholinergic and non-cholinergic cells, it should be noted that there are several limitations in electrophysiologically identifying true cholinergic cell type in vivo. Given that neurons are interconnected irrespective of cell type within the BF (Zaborszky and Duque, 2000, 2003; Zaborszky et al., 2012; Yang et al., 2014; $\mathrm{Xu}$ et al., 2015), it is possible that optically evoked responses may be observed even in non-cholinergic neurons. To avoid this confounding effect, one may take neurons with rapid onset responses as cholinergic neurons (Hangya et al., 2015; Xu et al., 2015). However, it is also known that some of cholinergic neurons indeed show delayed spiking (Unal et al., 2012), suggesting that a short light pulse (e.g., less than $100 \mathrm{~ms}$ ) may not be able to elicit spikes in cholinergic neurons. There is, therefore, a trade-off between false positive and negative, depending on the time window being assessed and the light pulse duration being used. Although we cannot exclude the possibility that there may have been false positive and negative in the present study, our observations are consistent with previous studies: firstly, the proportion of optogenetically identified cholinergic neurons
$(23 / 357=6.4 \%)$ is consistent with a stereological estimate ( 6.2\%) (Gritti et al., 2006). Secondly, we found that the spike width of cholinergic neurons significantly differs from that of non-cholinergic neurons, consistent with brain slice experiments (Unal et al., 2012). On the other hand, we are also aware that the proportion of cholinergic neurons is varied across reports (2-14\%) (Hangya et al., 2015; Xu et al., 2015). This discrepancy is probably due to light pulses (duration and power), ChR2 expression (virus or transgenic), optrode designs (wire or silicon probe) and sampled nuclei. To address this issue further, cell type specificity in the BF will need to be confirmed by other means in future, such as an imaging technology with high temporal resolution.

Basal forebrain populations showed lower spike count correlations, suggesting that BF cells fire more independently compared to cortical cells. Interestingly, optogenetically identified cholinergic neuron pairs show higher correlations. Given the small number of cholinergic neurons, this may be functionally reasonable in order to have an impact on the downstream as a population. On the other hand, inferring the underlying mechanisms of such higher correlations is not trivial: a recent comprehensive anatomical study indicates that the inputs to different cell types are similar (Do et al., 2016). Therefore, a subtle quantitative difference in long-range inputs and/or local connections may give rise to this cell typespecificity. In addition to further anatomical investigations, physiological and computational explorations will also be essential in addressing this issue, given the complexity of the biophysical mechanisms of correlated spiking (Renart et al., 2010; Mochol et al., 2015; Doiron et al., 2016). This is also the case for the mechanisms of higher correlations among slow rhythmic BF cells.

In the AC, we employed a conventional approach to classify cell classes based on spike waveforms (Sakata and Harris, 2009, 2012; Sakata, 2016). While it is likely that many of NS cells were parvalbumin-positive cells, BS cells likely consisted of heterogeneous cell classes, including not just excitatory cells, but also other inhibitory cell classes. This technical difficulty may have introduced discrepancies between the current results and previous reports (Sakata and Harris, 2012; Sakata, 2016). However, we confirmed a significant proportion of NS cells entrained to higher frequency bands, especially gamma bands, consistent with previous reports (Tremblay et al., 2016).

In the present study, we performed experiments under both anesthetized and unanesthetized conditions. Effects of condition were complex: Although we find a clear difference in frequency contents of cortical LFPs (Figure 2), neuronal activity at the single cell level provides a complex picture: spontaneous firing rate in unanesthetized $\mathrm{AC}$ is higher compared with that in anesthetized AC, consistent with previous reports (Sakata and Harris, 2009, 2012), whereas BF neuronal activity decreased in the unanesthetized condition. This BF activity may reflect diversity of state-dependent spontaneous firing across cell types (Zaborszky and Duque, 2003; Lee et al., 2005; Lin et al., 2006; Xu et al., 2015). In the BF, no statistically significant difference in rhythmic firing was found between conditions (Figure 6) whereas the AC showed a clear difference, that is, more AC cells showed rhythmic firing 
across a range of frequencies in the unanesthetized condition. These results support the notion that AC populations transmit signals on multiple timescales especially in the unanesthetized condition, and AC activity is more coordinated compared with BF population activity.

Why is AC population activity more orchestrated compared to $\mathrm{BF}$ population activity? Their anatomical organization may provide insights; although cortical neurons are highly heterogeneous, the cytoarchitecture in the cortex is highly modular (Szentagothai, 1983). On the other hand, the BF lacks such a cytoarchitecture. Accordingly, input and output organizations are also fundamentally different: in the cortex, incoming fibers are spatially organized and downstream targets also differ depending on cortical layers and cell types (Szentagothai, 1983; Linden and Schreiner, 2003; Douglas and Martin, 2004; Harris and Shepherd, 2015). In the BF, however, different cell types are intermingled and the anatomical organization is less modular (Zaborszky et al., 2015). These anatomical differences between both areas may help explain the underlying mechanisms of population activity in both structures. In addition to this, diversity of cortical inhibitory interneurons also plays a crucial role in the organization of cortical population activity (Tremblay et al., 2016). Compared with cortical interneurons, however, little is known about the local functional interactions in the $\mathrm{BF}$ in vivo, in particular functional roles of a diverse set of inhibitory neurons (Zaborszky and Duque, 2000; Zaborszky et al., 2012; Xu et al., 2015; Do et al., 2016). Therefore, further investigations are essential to mechanically understand the fundamental difference in the organization of population activity between the cortex and $\mathrm{BF}$.

Functionally, our results suggest that the operational timescale seems to be different between the BF and cortex. Although recent studies have appreciated fast actions of $\mathrm{BF}$ neurons (Sarter et al., 2009; Pinto et al., 2013; Hangya et al., 2015; Tingley et al., 2015), again the timescale is still slower than that in cortical operations, such as in a gamma range (tens of milliseconds). Our findings may also impose biophysical constraints on BF population activity for brain functions. For example, there is compelling evidence that gamma coherence plays a critical role in neuronal communications (Fries, 2005, 2015). However, this theory may not be applicable for BF populations. Rather our results support the hypothesis that $\mathrm{BF}$ populations play a role in enhancing the communication through gamma coherence in the cortex as BF parvalbumin-positive

\section{REFERENCES}

Anaclet, C., Pedersen, N. P., Ferrari, L. L., Venner, A., Bass, C. E., Arrigoni, E., et al. (2015). Basal forebrain control of wakefulness and cortical rhythms. Nat. Commun. 6:8744. doi: 10.1038/ncomms9744

Averbeck, B. B., Latham, P. E., and Pouget, A. (2006). Neural correlations, population coding and computation. Nat. Rev. Neurosci. 7, 358-366. doi: $10.1038 / \mathrm{nrn} 1888$

Ballinger, E. C., Ananth, M., Talmage, D. A., and Role, L. W. (2016). Basal forebrain cholinergic circuits and signaling in cognition and cognitive decline. Neuron 91, 1199-1218. doi: 10.1016/j.neuron.2016. 09.006 projection cells play a causal role in cortical gamma oscillations (Kim et al., 2015).

Are there any general principles of population activity across the $\mathrm{AC}$ and $\mathrm{BF}$ ? Although the structure of population activity in the $\mathrm{AC}$ and $\mathrm{BF}$ differs markedly, the skewed distribution of spontaneous firing rate is common across areas and states (Buzsaki and Mizuseki, 2014). Although technical caveats (e.g., sampling bias) have to be taken into account, the normal distribution should not be applied to model population activity in either structure. The difference in firing rate across cortical cell classes is also associated with anatomical and functional constraints as we have proposed previously (Sakata and Harris, 2009; Harris and Mrsic-Flogel, 2013). It may be conceivable that BF cells also show a similar tendency, which may be seen by analyzing different cell types more systematically. Another principle is the effect of correlated spiking on the downstream. Although the time scale differs between the BF and cortex, co-firing seems to have a larger impact onto the downstream. Thus, although much effort has been made recently in systematically elucidating cortical circuits (Kasthuri et al., 2015; Markram et al., 2015; Hawrylycz et al., 2016), comprehensive comparisons of population activity between different brain areas and cell types would provide further insights into the organizational principles of neural circuits.

\section{AUTHOR CONTRIBUTIONS}

JY and SS designed and conceived experiments. JY, TT, and SS performed electrophysiological and optogenetic experiments. JY performed histological experiments. JY and SS analyzed data. JY and SS wrote the manuscript with input from TT.

\section{FUNDING}

This work was supported by BBSRC (BB/K016830/1 and $\mathrm{BB} / \mathrm{M} 00905 \mathrm{X} / 1)$ to SS.

\section{ACKNOWLEDGMENTS}

We thank Christoph Kayser for his comments on an early version of the manuscript and Amisha Patel and Aimee Bias for their proofreading.

Barth, A. L., and Poulet, J. F. (2012). Experimental evidence for sparse firing in the neocortex. Trends Neurosci. 35, 345-355. doi: 10.1016/j.tins.2012.03.008

Buzsaki, G. (2010). Neural syntax: cell assemblies, synapsembles, and readers. Neuron 68, 362-385. doi: 10.1016/j.neuron.2010.09.023

Buzsaki, G., and Draguhn, A. (2004). Neuronal oscillations in cortical networks. Science 304, 1926-1929. doi: 10.1126/science.1099745

Buzsaki, G., and Mizuseki, K. (2014). The log-dynamic brain: how skewed distributions affect network operations. Nat. Rev. Neurosci. 15, 264-278. doi: $10.1038 / \mathrm{nrn} 3687$

Chavez, C., and Zaborszky, L. (2016). Basal forebrain cholinergic-auditory cortical network: primary versus nonprimary auditory cortical areas. Cereb. Cortex 27, 2335-2347. doi: 10.1093/cercor/bhw091 
Cohen, M. R., and Kohn, A. (2011). Measuring and interpreting neuronal correlations. Nat. Neurosci. 14, 811-819. doi: 10.1038/nn.2842

Curto, C., Sakata, S., Marguet, S., Itskov, V., and Harris, K. D. (2009). A simple model of cortical dynamics explains variability and state dependence of sensory responses in urethane-anesthetized auditory cortex. J. Neurosci. 29, 10600-10612. doi: 10.1523/JNEUROSCI.2053-09.2009

Do, J. P., Xu, M., Lee, S. H., Chang, W. C., Zhang, S., Chung, S., et al. (2016). Cell type-specific long-range connections of basal forebrain circuit. Elife 5:e13214. doi: $10.7554 /$ eLife. 13214

Doiron, B., Litwin-Kumar, A., Rosenbaum, R., Ocker, G. K., and Josic, K. (2016). The mechanics of state-dependent neural correlations. Nat. Neurosci. 19, 383-393. doi: $10.1038 / \mathrm{nn} .4242$

Douglas, R. J., and Martin, K. A. (2004). Neuronal circuits of the neocortex. Annu. Rev. Neurosci. 27, 419-451. doi: 10.1146/annurev.neuro.27.070203.144152

Eggermann, E., Kremer, Y., Crochet, S., and Petersen, C. C. (2014). Cholinergic signals in mouse barrel cortex during active whisker sensing. Cell Rep. 9, 1654-1660. doi: 10.1016/j.celrep.2014.11.005

Everitt, B. J., and Robbins, T. W. (1997). Central cholinergic systems and cognition. Annu. Rev. Psychol. 48, 649-684. doi: 10.1146/annurev.psych.48.1.649

Fries, P. (2005). A mechanism for cognitive dynamics: neuronal communication through neuronal coherence. Trends Cogn. Sci. 9, 474-480. doi: 10.1016/j.tics. 2005.08.011

Fries, P. (2015). Rhythms for cognition: communication through coherence. Neuron 88, 220-235. doi: 10.1016/j.neuron.2015.09.034

Froemke, R. C., Merzenich, M. M., and Schreiner, C. E. (2007). A synaptic memory trace for cortical receptive field plasticity. Nature 450, 425-429. doi: 10.1038/ nature 06289

Gritti, I., Henny, P., Galloni, F., Mainville, L., Mariotti, M., and Jones, B. E. (2006). Stereological estimates of the basal forebrain cell population in the rat, including neurons containing choline acetyltransferase, glutamic acid decarboxylase or phosphate-activated glutaminase and colocalizing vesicular glutamate transporters. Neuroscience 143, 1051-1064. doi: 10.1016/j.neuroscience.2006. 09.024

Grün, S. (2009). Data-driven significance estimation for precise spike correlation. J. Neurophysiol. 101, 1126-1140. doi: 10.1152/jn.00093.2008

Hangya, B., Ranade, S. P., Lorenc, M., and Kepecs, A. (2015). Central cholinergic neurons are rapidly recruited by reinforcement feedback. Cell 162, 1155-1168. doi: 10.1016/j.cell.2015.07.057

Harris, K. D., Henze, D. A., Csicsvari, J., Hirase, H., and Buzsaki, G. (2000). Accuracy of tetrode spike separation as determined by simultaneous intracellular and extracellular measurements. J. Neurophysiol. 84, 401-414.

Harris, K. D., and Mrsic-Flogel, T. D. (2013). Cortical connectivity and sensory coding. Nature 503, 51-58. doi: 10.1038/nature12654

Harris, K. D., and Shepherd, G. M. (2015). The neocortical circuit: themes and variations. Nat. Neurosci. 18, 170-181. doi: 10.1038/nn.3917

Harrison, T. C., Pinto, L., Brock, J. R., and Dan, Y. (2016). Calcium imaging of basal forebrain activity during innate and learned behaviors. Front. Neural Circuits 10:36. doi: 10.3389/fncir.2016.00036

Hasselmo, M. E., and Sarter, M. (2011). Modes and models of forebrain cholinergic neuromodulation of cognition. Neuropsychopharmacology 36, 52-73. doi: $10.1038 /$ npp. 2010.104

Hawrylycz, M., Anastassiou, C., Arkhipov, A., Berg, J., Buice, M., Cain, N., et al. (2016). Inferring cortical function in the mouse visual system through largescale systems neuroscience. Proc. Natl. Acad. Sci. U.S.A. 113, 7337-7344. doi: 10.1073/pnas.1512901113

Henze, D. A., Borhegyi, Z., Csicsvari, J., Mamiya, A., Harris, K. D., and Buzsaki, G. (2000). Intracellular features predicted by extracellular recordings in the hippocampus in vivo. J. Neurophysiol. 84, 390-400.

Hirase, H., Leinekugel, X., Czurko, A., Csicsvari, J., and Buzsaki, G. (2001). Firing rates of hippocampal neurons are preserved during subsequent sleep episodes and modified by novel awake experience. Proc. Natl. Acad. Sci. U.S.A. 98, 9386-9390. doi: 10.1073/pnas.161274398

Hromadka, T., Deweese, M. R., and Zador, A. M. (2008). Sparse representation of sounds in the unanesthetized auditory cortex. PLOS Biol. 6:e16. doi: 10.1371/ journal.pbio.0060016

Kalmbach, A., Hedrick, T., and Waters, J. (2012). Selective optogenetic stimulation of cholinergic axons in neocortex. J. Neurophysiol. 107, 2008-2019. doi: $10.1152 /$ jn. 00870.2011
Kasthuri, N., Hayworth, K. J., Berger, D. R., Schalek, R. L., Conchello, J. A., Knowles-Barley, S., et al. (2015). Saturated reconstruction of a volume of neocortex. Cell 162, 648-661. doi: 10.1016/j.cell.2015.06.054

Kayser, C., Wilson, C., Safaai, H., Sakata, S., and Panzeri, S. (2015). Rhythmic auditory cortex activity at multiple timescales shapes stimulus-response gain and background firing. J. Neurosci. 35, 7750-7762. doi: 10.1523/JNEUROSCI. 0268-15.2015

Kilgard, M. P., and Merzenich, M. M. (1998). Cortical map reorganization enabled by nucleus basalis activity. Science 279, 1714-1718.

Kim, T., Thankachan, S., Mckenna, J. T., Mcnally, J. M., Yang, C., Choi, J. H., et al. (2015). Cortically projecting basal forebrain parvalbumin neurons regulate cortical gamma band oscillations. Proc. Natl. Acad. Sci. U.S.A. 112, 3535-3540. doi: 10.1073/pnas.1413625112

Lee, M. G., Hassani, O. K., Alonso, A., and Jones, B. E. (2005). Cholinergic basal forebrain neurons burst with theta during waking and paradoxical sleep. J. Neurosci. 25, 4365-4369. doi: 10.1523/JNEUROSCI.0178-05. 2005

Lin, S. C., Brown, R. E., Hussain Shuler, M. G., Petersen, C. C., and Kepecs, A. (2015). optogenetic dissection of the basal forebrain neuromodulatory control of cortical activation, plasticity, and cognition. J. Neurosci. 35, 13896-13903. doi: 10.1523/JNEUROSCI.2590-15.2015

Lin, S. C., Gervasoni, D., and Nicolelis, M. A. (2006). Fast modulation of prefrontal cortex activity by basal forebrain noncholinergic neuronal ensembles. J. Neurophysiol. 96, 3209-3219. doi: 10.1152/jn.00524.2006

Lin, S. C., and Nicolelis, M. A. (2008). Neuronal ensemble bursting in the basal forebrain encodes salience irrespective of valence. Neuron 59, 138-149. doi: 10.1016/j.neuron.2008.04.031

Linden, J. F., and Schreiner, C. E. (2003). Columnar transformations in auditory cortex? A comparison to visual and somatosensory cortices. Cereb. Cortex 13, $83-89$.

Litwin-Kumar, A., and Doiron, B. (2012). Slow dynamics and high variability in balanced cortical networks with clustered connections. Nat. Neurosci. 15, 1498-1505. doi: 10.1038/nn.3220

Luczak, A., Bartho, P., Marguet, S. L., Buzsaki, G., and Harris, K. D. (2007). Sequential structure of neocortical spontaneous activity in vivo. Proc. Natl. Acad. Sci. U.S.A. 104, 347-352. doi: 10.1073/pnas.0605643104

Madisen, L., Mao, T., Koch, H., Zhuo, J. M., Berenyi, A., Fujisawa, S., et al. (2012). A toolbox of Cre-dependent optogenetic transgenic mice for lightinduced activation and silencing. Nat. Neurosci. 15, 793-802. doi: 10.1038/nn. 3078

Markram, H., Muller, E., Ramaswamy, S., Reimann, M. W., Abdellah, M., Sanchez, C. A., et al. (2015). Reconstruction and simulation of neocortical microcircuitry. Cell 163, 456-492. doi: 10.1016/j.cell.2015.09.029

McAlinden, N., Gu, E., Dawson, M. D., Sakata, S., and Mathieson, K. (2015). Optogenetic activation of neocortical neurons in vivo with a sapphire-based micro-scale LED probe. Front. Neural Circuits 9:25. doi: 10.3389/fncir.2015. 00025

McAlinden, N., Massoubre, D., Richardson, E., Gu, E., Sakata, S., Dawson, M. D., et al. (2013). Thermal and optical characterization of micro-LED probes for in vivo optogenetic neural stimulation. Opt. Lett. 38, 992-994. doi: 10.1364/OL. 38.000992

McKenna, J. T., Yang, C., Franciosi, S., Winston, S., Abarr, K. K., Rigby, M. S. et al. (2013). Distribution and intrinsic membrane properties of basal forebrain GABAergic and parvalbumin neurons in the mouse. J. Comp. Neurol. 521, 1225-1250. doi: 10.1002/cne.23290

Mesulam, M. M., Mufson, E. J., Wainer, B. H., and Levey, A. I. (1983). Central cholinergic pathways in the rat: an overview based on an alternative nomenclature (Ch1-Ch6). Neuroscience 10, 1185-1201.

Middleton, J. W., Omar, C., Doiron, B., and Simons, D. J. (2012). Neural correlation is stimulus modulated by feedforward inhibitory circuitry. J. Neurosci. 32, 506-518. doi: 10.1523/JNEUROSCI.3474-11.2012

Mizuseki, K., and Buzsaki, G. (2013). Preconfigured, skewed distribution of firing rates in the hippocampus and entorhinal cortex. Cell Rep. 4, 1010-1021. doi: 10.1016/j.celrep.2013.07.039

Mochol, G., Hermoso-Mendizabal, A., Sakata, S., Harris, K. D., and De La Rocha, J. (2015). Stochastic transitions into silence cause noise correlations in cortical circuits. Proc. Natl. Acad. Sci. U.S.A. 112, 3529-3534. doi: 10.1073/pnas. 1410509112 
Nelson, A., and Mooney, R. (2016). The basal forebrain and motor cortex provide convergent yet distinct movement-related inputs to the auditory cortex. Neuron 90, 635-648. doi: 10.1016/j.neuron.2016.03.031

O'Connor, D. H., Peron, S. P., Huber, D., and Svoboda, K. (2010). Neural activity in barrel cortex underlying vibrissa-based object localization in mice. Neuron 67, 1048-1061. doi: 10.1016/j.neuron.2010.08.026

Okun, M., Yger, P., Marguet, S. L., Gerard-Mercier, F., Benucci, A., Katzner, S., et al. (2012). Population rate dynamics and multineuron firing patterns in sensory cortex. J. Neurosci. 32, 17108-17119. doi: 10.1523/JNEUROSCI.1831-12.2012

Peyrache, A., Dehghani, N., Eskandar, E. N., Madsen, J. R., Anderson, W. S., Donoghue, J. A., et al. (2012). Spatiotemporal dynamics of neocortical excitation and inhibition during human sleep. Proc. Natl. Acad. Sci. U.S.A. 109, 1731-1736. doi: 10.1073/pnas.1109895109

Pinto, L., Goard, M. J., Estandian, D., Xu, M., Kwan, A. C., Lee, S. H., et al. (2013). Fast modulation of visual perception by basal forebrain cholinergic neurons. Nat. Neurosci. 16, 1857-1863. doi: 10.1038/nn.3552

Renart, A., De La Rocha, J., Bartho, P., Hollender, L., Parga, N., Reyes, A., et al. (2010). The asynchronous state in cortical circuits. Science 327, 587-590.

Reyes-Puerta, V., Yang, J. W., Siwek, M. E., Kilb, W., Sun, J. J., and Luhmann, H. J. (2016). Propagation of spontaneous slow-wave activity across columns and layers of the adult rat barrel cortex in vivo. Brain Struct. Funct. 221, 4429-4449. doi: 10.1007/s00429-015-1173-x

Rossant, C., Kadir, S. N., Goodman, D. F., Schulman, J., Hunter, M. L., Saleem, A. B., et al. (2016). Spike sorting for large, dense electrode arrays. Nat. Neurosci. 19, 634-641. doi: 10.1038/nn.4268

Sakata, S. (2016). State-dependent and cell type-specific temporal processing in auditory thalamocortical circuit. Sci. Rep. 6:18873. doi: 10.1038/srep 18873

Sakata, S., and Harris, K. D. (2009). Laminar structure of spontaneous and sensory-evoked population activity in auditory cortex. Neuron 64, 404-418. doi: 10.1016/j.neuron.2009.09.020

Sakata, S., and Harris, K. D. (2012). Laminar-dependent effects of cortical state on auditory cortical spontaneous activity. Front. Neural Circuits 6:109. doi: 10.3389/fncir.2012.00109

Sarter, M., Parikh, V., and Howe, W. M. (2009). Phasic acetylcholine release and the volume transmission hypothesis: time to move on. Nat. Rev. Neurosci. 10, 383-390. doi: 10.1038/nrn2635

Scharf, R., Tsunematsu, T., Mcalinden, N., Dawson, M. D., Sakata, S., and Mathieson, K. (2016). Depth-specific optogenetic control in vivo with a scalable, high-density muLED neural probe. Sci. Rep. 6:28381. doi: 10.1038/srep28381

Schmitzer-Torbert, N., Jackson, J., Henze, D., Harris, K., and Redish, A. D. (2005). Quantitative measures of cluster quality for use in extracellular recordings. Neuroscience 131, 1-11.

Schroeder, C. E., and Lakatos, P. (2009). Low-frequency neuronal oscillations as instruments of sensory selection. Trends Neurosci. 32, 9-18. doi: 10.1016/j.tins. 2008.09.012

Steriade, M. (2001). Impact of network activities on neuronal properties in corticothalamic systems. J. Neurophysiol. 86, 1-39.

Steriade, M., Nunez, A., and Amzica, F. (1993). A novel slow ( $<1 \mathrm{~Hz}$ ) oscillation of neocortical neurons in vivo: depolarizing and hyperpolarizing components. J. Neurosci. 13, 3252-3265.

Stujenske, J. M., Spellman, T., and Gordon, J. A. (2015). Modeling the spatiotemporal dynamics of light and heat propagation for In Vivo optogenetics. Cell Rep. 12, 525-534. doi: 10.1016/j.celrep.2015.06.036
Szentagothai, J. (1983). The modular architectonic principle of neural centers. Rev. Physiol. Biochem. Pharmacol. 98, 11-61.

Tingley, D., Alexander, A. S., Quinn, L. K., Chiba, A. A., and Nitz, D. A. (2015). Cell assemblies of the basal forebrain. J. Neurosci. 35, 2992-3000. doi: 10.1523/ JNEUROSCI.4432-14.2015

Tremblay, R., Lee, S., and Rudy, B. (2016). GABAergic interneurons in the neocortex: from cellular properties to circuits. Neuron 91, 260-292. doi: 10.1016/j.neuron.2016.06.033

Unal, C. T., Golowasch, J. P., and Zaborszky, L. (2012). Adult mouse basal forebrain harbors two distinct cholinergic populations defined by their electrophysiology. Front Behav. Neurosci. 6:21. doi: 10.3389/fnbeh.2012.00021

Weinberger, N. M. (2004). Specific long-term memory traces in primary auditory cortex. Nat. Rev. Neurosci. 5, 279-290. doi: 10.1038/nrn1366

Whitehouse, P. J., Price, D. L., Struble, R. G., Clark, A. W., Coyle, J. T., and Delon, M. R. (1982). Alzheimer's disease and senile dementia: loss of neurons in the basal forebrain. Science 215, 1237-1239.

Woolf, N. J. (1991). Cholinergic systems in mammalian brain and spinal cord. Prog. Neurobiol. 37, 475-524.

Xu, M., Chung, S., Zhang, S., Zhong, P., Ma, C., Chang, W. C., et al. (2015). Basal forebrain circuit for sleep-wake control. Nat. Neurosci. 18, 1641-1647. doi: $10.1038 / \mathrm{nn} .4143$

Yang, C., Mckenna, J. T., Zant, J. C., Winston, S., Basheer, R., and Brown, R. E. (2014). Cholinergic neurons excite cortically projecting basal forebrain GABAergic neurons. J. Neurosci. 34, 2832-2844. doi: 10.1523/JNEUROSCI. 3235-13.2014

Zaborszky, L., Csordas, A., Mosca, K., Kim, J., Gielow, M. R., Vadasz, C., et al. (2015). Neurons in the basal forebrain project to the cortex in a complex topographic organization that reflects corticocortical connectivity patterns: an experimental study based on retrograde tracing and 3D reconstruction. Cereb. Cortex 25, 118-137. doi: 10.1093/cercor/bht210

Zaborszky, L., and Duque, A. (2000). Local synaptic connections of basal forebrain neurons. Behav. Brain Res. 115, 143-158.

Zaborszky, L., and Duque, A. (2003). Sleep-wake mechanisms and basal forebrain circuitry. Front. Biosci. 8:d1146-d1169.

Zaborszky, L., Van Del Pol, A., and Gyengesi, E. (2012). "The basal forebrain cholinergic projection system in mice," in The Mouse Nervous System, eds C. Watson, G. Paxinos, and L. Puelles (Amsterdam: Elsevier), 684-718.

Zant, J. C., Kim, T., Prokai, L., Szarka, S., Mcnally, J., Mckenna, J. T., et al. (2016). Cholinergic neurons in the basal forebrain promote wakefulness by actions on neighboring non-cholinergic neurons: an opto-dialysis study. J. Neurosci. 36, 2057-2067. doi: 10.1523/JNEUROSCI.3318-15.2016

Conflict of Interest Statement: The authors declare that the research was conducted in the absence of any commercial or financial relationships that could be construed as a potential conflict of interest.

Copyright (c) 2017 Yague, Tsunematsu and Sakata. This is an open-access article distributed under the terms of the Creative Commons Attribution License (CC BY). The use, distribution or reproduction in other forums is permitted, provided the original author(s) or licensor are credited and that the original publication in this journal is cited, in accordance with accepted academic practice. No use, distribution or reproduction is permitted which does not comply with these terms. 